\title{
Timeless Approach to Quantum Jumps
}

\author{
Ignazio Licata ${ }^{1,2}$ \& Leonardo Chiatti ${ }^{3}$ \\ 1 ISEM Institute for Scientific Methodology, Palermo, Italy. E-mail: ignazio.licata@ejtp.info \\ ${ }^{2}$ School of Advanced International Studies on Applied Theoretical and Nonlinear Methodologies in Physics, Bari, Italy \\ ${ }^{3}$ AUSL VT Medical Physics Laboratory, Via Enrico Fermi 15, Viterbo, Italy. E-mail: leonardo.chiatti@asl.vt.it
}

Editors: Eliahu Cohen \& John Ashmead

Article history: Submitted on November 7, 2014; Accepted on August 16, 2015; Published on October 11, 2015.

\section{A} ccording to the usual quantum description, the time evolution of the quantum state is continuous and deterministic except when a discontinuous and indeterministic collapse of state vector occurs. The collapse has been a central topic since the origin of the theory, although there are remarkable theoretical proposals to understand its nature, such as the Ghirardi-Rimini-Weber. Another possibility could be the assimilation of collapse with the now experimentally well established phenomenon of quantum jump, postulated by Bohr already in 1913. The challenge of nonlocality offers an opportunity to reconsider the quantum jump as a fundamental element of the logic of the physical world, rather than a subsidiary accident. We propose here a simple preliminary model that considers quantum jumps as processes of entry to and exit from the usual temporal domain to a timeless vacuum, without contradicting the quantum relativistic formalism, and we present some potential connections with particle physics. Quanta 2015; 4: 10-26.

(c) (1) This is an open access article distributed under the terms of the Creative Commons Attribution License CC-BY-3.0, which permits unrestricted use, distribution, and reproduction in any medium, provided the original author and source are credited.

\section{Introduction}

During the first phase of the development of quantum theory (1913-1927) three fundamental questions were posed: 1) the quantization of material motion, represented by discrete electron orbits; 2) the quantization of the field, in terms of the hypothesis of the emission and absorption of photons; and 3) the discontinuity of motion, represented by quantum jumps between stationary orbits. With regard to points 1) and 2), the subsequent definition of quantum formalism led to quantum mechanics and quantum field theory, or, in other words, respectively to first and second quantization. It has been noted that quantum mechanics and quantum field theory still do not constitute an organic structure; wave-particle duality in particular, which proved so useful for the description of Einstein-Podolsky-Rosen-Bell phenomena, has no place in descriptions based strictly on quantum field theory [1].

The point 3 ) did not bring any particular development; it is still the source of a periodically renewing debate, in particular about the questions related to the wavefunction collapse - the anomalous postulate of quantum mechanics - and its incompatibility with special relativity [1]. The concept of quantum jump is closely connected to the wavefunction collapse: the decay of an atom which transits to its ground state is both a quantum jump and the event of the collapse of the wavefunction associated to the state of that atom. The actual manifestation of this kind of events can be easily showed, for example through the detection of the photon emitted (if the transition is radia- 
tive). Various theoretical proposals contributed to get the collapse irreducibility into perspective, bringing it back, at least partially, to the dynamics of the system; let us remember here the results of the Milan-Pavia School, the Bohm-Bub theory, the Ghirardi-Rimini-Weber dynamical reduction program and decoherent histories [2-6]. On the experimental side, since the end of the 1980s, new technologies have allowed extraordinary realizations in revealing single quantum jumps in any kind of quantum system, so confirming and extending the original 1913 Bohr intuition. Nowadays, the direct observation of quantum jumps has been widely confirmed for trapped atoms and ions [7-.9], single molecules [10], photons [11], single electrons in cyclotron [12], Josephson junctions [13], nuclear [14] and electronic [15] spin, superconducting cavities [16] thus providing an impressive demonstration of the helpful Bohr's intuition. The initial hesitancy about the real existence of quantum jumps, in particular by the community of quantum optics, is now only a distant memory of long time ago [17]. In conclusion, in the quantum jump the undulatory ubiquity of a quantum object (or state) and its particle-like localized aspect meet in a collapse event. So, we expect that a new theory about quantum jumps can tell us something on the nature of a quantum entity [18-20].

In his classical book on quantum mechanical principles [21], Heisenberg delineates with his usual clarity two possible ways to build quantum mechanics, both of which were coherent with the uncertainty principle: the first, a space-time description tending to save the classical concepts by means of a statistical connection; the second, adopting a pre-spatial mathematical scheme and a different concept of causality. In the 1930s there was no cogent reason to choose the second way. Nowadays, quantum nonlocality offers a valid reason to explore a different approach where the nonlocal features of quantum mechanics are not necessarily restricted to the entangled amplitudes, but are instead connected to a time-reversal approach [22], or some timeless background.

In this paper we propose a model for the discontinuous evolution of the quantum amplitude associated to a system: the so-called quantum jump. We assume here the objective nature of the wavefunction collapse that is actually identified with a quantum jump associated to a real micro-interaction. We define an explanatory model, by broadening and redefining the quantum field theory vacuum with the introduction of a complex time that regulates the structure of interaction vertices in real time as measured by an observer. We discuss a thermalized vacuum in terms of the relationship between imaginary time and temperature. The strategy of introducing imaginary time is well-known in cosmology and field theory, and has been proven effective in the removal of singularities and the treatment of deterministic fields perturbed by an appropriate stochastic noise [23-26]. In this context, it is equivalent to a description of the vacuum as a structure of relationships between complex events characterized by a fundamental time scale, which replaces the standard quantum mechanical concept of a particle. The particle concept appears as still less credible after recent experiments where packets of physical quantities related to the same particle are manifested along different paths within an interferometer [27].

The theoretical choice adopted in this paper aims to avoid the ambiguities about the concept of quantum jump connected to the semi-classical nature of the waveparticle dualism. Our line of reasoning follows the transactional interpretation of quantum mechanics which sees the generic quantum system as a network of micro-events [28] and is thus closer to the spirit of quantum field theory. In this sense, we can agree with D. Zeh ("nor are there particles!") [29] yet not dismissing the idea of quantum jump, which is here reformulated within a new theoretical frame. Some potential applications to particle physics are even shortly outlined.

\section{Clicks and interaction vertices}

Let us consider a prepared system, at time 0 , in a physical state associated with $\left|A_{0}\right\rangle$. We assume that in the interval $[0, t]$ the ket associated with the state of the system evolves under the effect of a unitary time evolution operator $S$, and that $\left|A_{t}\right\rangle=S(t, 0)\left|A_{0}\right\rangle$. At instant $t$, in response to an interaction, the system abruptly transits to the state represented by $|B\rangle$. We could say that the system prepared in the initial state $A_{0}$ is detected in the final state $B$. This is also the initial state, associated with ket $|B\rangle$, of a new preparation-detection pair. The full model can be understood by reading the following line from right to left: $|B\rangle\left\langle B|S(t, 0)| A_{0}\right\rangle$, in which a unitary evolution represented by the amplitude $\left\langle B|S(t, 0)| A_{0}\right\rangle=\left\langle B \mid A_{t}\right\rangle$ is concatenated with a quantum jump $|B\rangle\langle B|$. By way of example, let $A_{0}$ be the state of a particle beam emitted by a source, $A_{t}$ the state of the same beam incident on a screen with a single slit, $B$ the state of the beam selected by the slit and diffracted beyond the screen. The bra portion $\langle B|$ of the quantum jump is turned toward the past light cone and closes the previous unitary propagation; the ket portion $|B\rangle$ is directed toward the future light cone and opens a new segment of unitary evolution.

Naturally, the entire process is symmetrical in time and can be read in reverse; thus bra $\langle B|$ becomes the initial condition of the time evolution described by the evolution operator $S^{-1}=S^{+}$, which ends with the final condition represented by ket $\left|A_{0}\right\rangle$. 
There is no substantial difference in the application of this model in quantum mechanics and in quantum field theory, except that in quantum field theory it is applied to elementary particles and combinations of their creation and annihilation operators. For example, $|B\rangle\langle B|$ could represent an interaction vertex between particles and $|B\rangle$ the creation of a set of particles exiting from it (we suppose that $|B\rangle\langle B|$ acts on its right). It should be understood, however, that this is a real interaction vertex with real particles. Virtual interactions and virtual particles are aspects of the expansion of the operator $S$ into partial amplitudes and have no physical reality in this context. From this perspective, therefore, a quantum jump is synonymous with a micro-event or click. In this paper we aim to present a simple basic physical model for jumps, represented by the projector $|B\rangle\langle B|$. A click is considered to consist of two simultaneous (semi-) events: the destruction of the ingoing state $B$ and the creation of the outgoing state $B$. We assume a jump is an interaction vertex between elementary particles and $B$ is the state of the particles leaving the vertex.

The emphasis is therefore placed on interaction vertices and particles are considered as links between these vertices. Vertices and links form a network of relationships. This position is consistent with other theoretical proposals; for example, with the transactional interpretation, which is an interesting attempt to create a unified language to describe quantum phenomena [30-33].

In quantum formalism, the state of a many particle system is represented by a mixture of several states which are themselves entanglements of single particle states. The actualization of one of these single particle states corresponds to the selection, in the superposition that represents the entanglement, of the term wherein it appears and the consequent readjustment of the coefficients of the density matrix associated with the mixture. In an attempt to develop a model of the actualization process it is therefore necessary to focus on the single particle states, because the actualization at a higher level is a consequence of the actualization of the single particle states. On the other hand, the actualization of single particle state ensues from the interaction of the particle with material elements (measurement devices, etc.) which in turn consist of particles. We must therefore consider the single particle states entering a real interaction vertex, and those exiting from it. Both the former and the latter may be entangled.

We consider the action of the operators $|A\rangle\langle A|| B\rangle,\langle B|$ on the single particle state $|\Psi\rangle=c_{A}|A\rangle+c_{B}|B\rangle$, with $c_{A} c_{A}^{*}+c_{B} c_{B}^{*}=1,|A\rangle=\left|A_{1}\right\rangle+\left|A_{2}\right\rangle+\ldots+\left|A_{n}\right\rangle$.

The action of $|B\rangle\langle B|$ will, by assumption, correspond to the localization of the particle in time (i.e. the event of its emission or absorption). Following this action, the particle will remain delocalized in accordance with the wavefunction $\langle x \mid B\rangle$, which will not necessarily be a Dirac $\delta$ in the $x$ position coordinates. In other words, the event $|B\rangle\langle B|$ does not necessarily imply a maximally precise spatial localization of the particle and is therefore aspatial.

The action of $|A\rangle\langle A|$ shall not correspond to any particle localization, i.e. it will not be associated with any emission or absorption of the same. It will preserve the phase relation between the states $\left|A_{i}\right\rangle(i=1,2, \ldots)$. The event $|A\rangle\langle A|$ leaves the particle delocalized according to the wavefunction $\langle x \mid A\rangle$, and will therefore be aspatial. That said, we will call quantum jump the event $|B\rangle\langle B|$ that begins or ends a segment of unitary time evolution of the particle state. The event $|A\rangle\langle A|$ on the other hand preserves the unitary nature of the evolution. To better understand the relationship between quantum jump and unitary evolution, we consider three distinct examples.

a) Reflection from a specular surface. A particle incident on a perfectly reflecting surface devoid of absorption is subject to the action of the sole operator $|A\rangle\langle A|$, where $|A\rangle$ is the reflected state. If $|\Psi\rangle$ is the incident state, $\langle A \mid \Psi\rangle$ is the amplitude of the reflected state. The reflection will occur over the entire surface of the mirror, thus the states $\left|A_{i}\right\rangle$ may be the states reflected by various points of the mirror, labelled by the (continuous) index $i$. Because there is no localized reflection $\left|A_{i}\right\rangle\left\langle A_{i}\right|$, the phase relation between the several states $\left|A_{i}\right\rangle$ is preserved. No quantum jump occurs and the particle state evolution remains unitary.

b) Impact on an opaque screen with double slit. A particle incident on the screen has two possibilities: either it is absorbed by the screen (event $|B\rangle\langle B|$ ) or it goes through the double slit (event $|A\rangle\langle A|)$. In the first case, the absorption occurs at a precise moment in time and has the effect of destroying the particle (for example, a photon) or of localizing it in the spatial volume corresponding to its new state (for example, atomic capture of an electron). $|B\rangle$ is in the first case the electromagnetic vacuum state of quantum electrodynamics, in the second case it is the atomic orbital of the captured electron. If the incident particle is transmuted into other particles, $|B\rangle$ will be the initial state of these particles. In the case where the event $|A\rangle\langle A|$ occurs, the states $\left|A_{i}\right\rangle(i=1,2)$ are those relative to crossing slit 1 or slit 2 respectively. These two states are in phase relation, because no process of localized crossing $\left|A_{i}\right\rangle\left\langle A_{i}\right|$ occurs and the evolution of the state $|A\rangle$ formed by their superposition remains unitary. In this case as well, the particle is delocalized according to the new wavefunction $\langle x \mid A\rangle$ (Fig. 1).

c) Atomic decay. Let us consider a two-level atom: the ground level $|B\rangle$ and the excited level $|A\rangle$. The atom is prepared in $|A\rangle$ at time 0 and the subsequent unitary evolution 


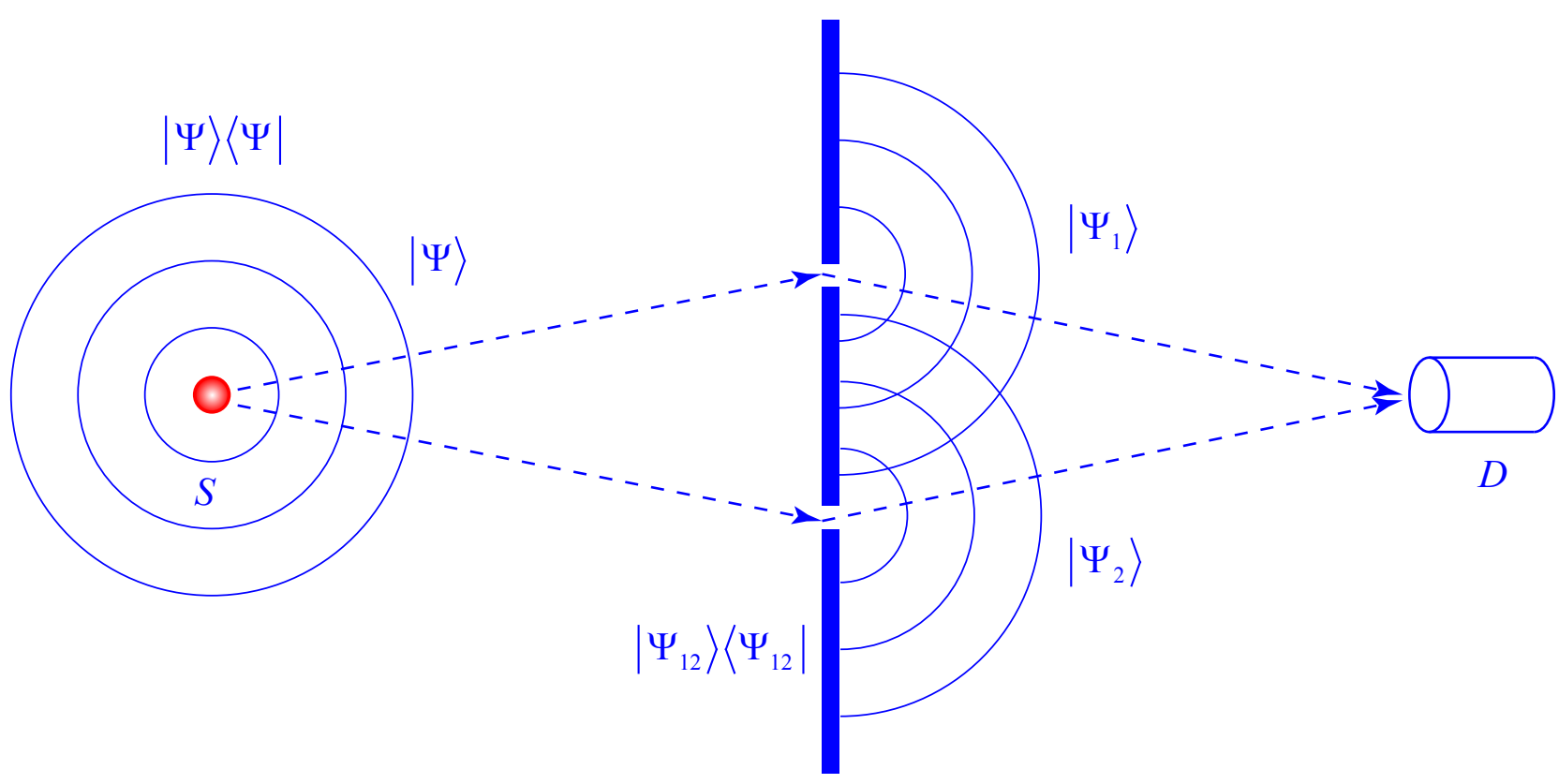

Figure 1: The double slit experiment. The action of the double slit upon $|\Psi\rangle$ is non-unitary with respect to the absorption. However, the aspatial reduction event $\left|\Psi_{12}\right\rangle\left\langle\Psi_{12}\right|$ maintains the phase coherence of $\left|\Psi_{1}\right\rangle$ and $\left|\Psi_{2}\right\rangle$ components according to the usual path integral formalism. Legend: $S=$ source, $D=$ detector, $\left|\Psi_{12}\right\rangle=\left|\Psi_{1}\right\rangle+\left|\Psi_{2}\right\rangle$.

of its state produces the superposition $|\Psi(t)\rangle=\alpha|A\rangle+\beta|B\rangle$. If we verify the state of the atom at a certain time $t_{0}$ we have two possibilities: either the atom has decayed (i.e., $|B\rangle\langle B|$ has acted at a given time $t$ with $\left.0<t<t_{0}\right)$, or it has not decayed (i.e. $|B\rangle\langle B|$ has not acted at any given time $t$ with $0<t<t_{0}$ ). In the first case, the verification action is equivalent to destroying the previous state $|B\rangle$ and recreating it, and it is therefore expressed as the projector $|B\rangle\langle B|$; in the second case, it is equivalent to acting with $|A\rangle\langle A|$ on the superposition $\left|\Psi\left(t_{0}\right)\right\rangle$, reinitializing its unitary evolution. In both cases, the verification action corresponds to a physical process represented by a quantum jump. It is important not to confuse this jump with that possibly occurring in the interval $0<t<t_{0}$ and completed autonomously by the atom coupled with the electromagnetic field (Fig. 2).

These examples clarify the boundary between the unitary evolution of the quantum state and the quantum jump phenomenon. The latter is always associated with the precise localization of the particle over time (although not necessarily in space) and therefore its emission or absorption, or with the restart of its state. We have indeed seen cases of absorption on a screen, of emission by an atom which decays or of restart of a superposition of atomic states. The quantum jump may correspond to an observation-measurement process (as in the verification of example c), or not (as in the case of absorption on the screen in example $b$ or of the spontaneous decay of the atom in example c). Thus, the state reduction ensuing from a projective measurement process should be considered as a particular example of the more general concept of quantum jump. We propose to stop thinking in terms of persistent particles and their states, even though so far we conformed to this language by convention, but rather in terms of clicks bi-oriented in time, with reference to the projectors $|B\rangle\langle B|$ associated with the quantum jumps.

The time symmetry of a click, which appears as a kind of two-faced Janus along the time line, has several equivalents in quantum physics. For example, we can split the $x(t)$ coordinate of a quantum object into two coordinates, $x_{+}(t)$ (forward) and $x_{-}(t)$ (backward) using the Wigner-Feynman distribution, which incorporates nonlocal aspects of quantum mechanics [34, 35]. The evolution of the density matrix associated with this distribution leads to two copies of the Schrödinger equation, backward and forward, controlled by two Hamiltonian operators, yielding the Bohr frequency transitions. This doubling of the degrees of freedom $\left(x_{ \pm}, p_{ \pm}\right)$also occurs in dissipative quantum field theory and is therefore a fundamental structural aspect of both quantum mechanics and quantum field theory [36-40].

In the next sections we provide an in-depth description of what happens in a single quantum jump $|B\rangle\langle B|$. We will try to clarify the non-unitary aspects of the real interaction between elementary particles, not described by the unitary evolution of the state vector. For this purpose, we have to add new concepts to current quantum formalism, in order to specify the collapse postulate. 


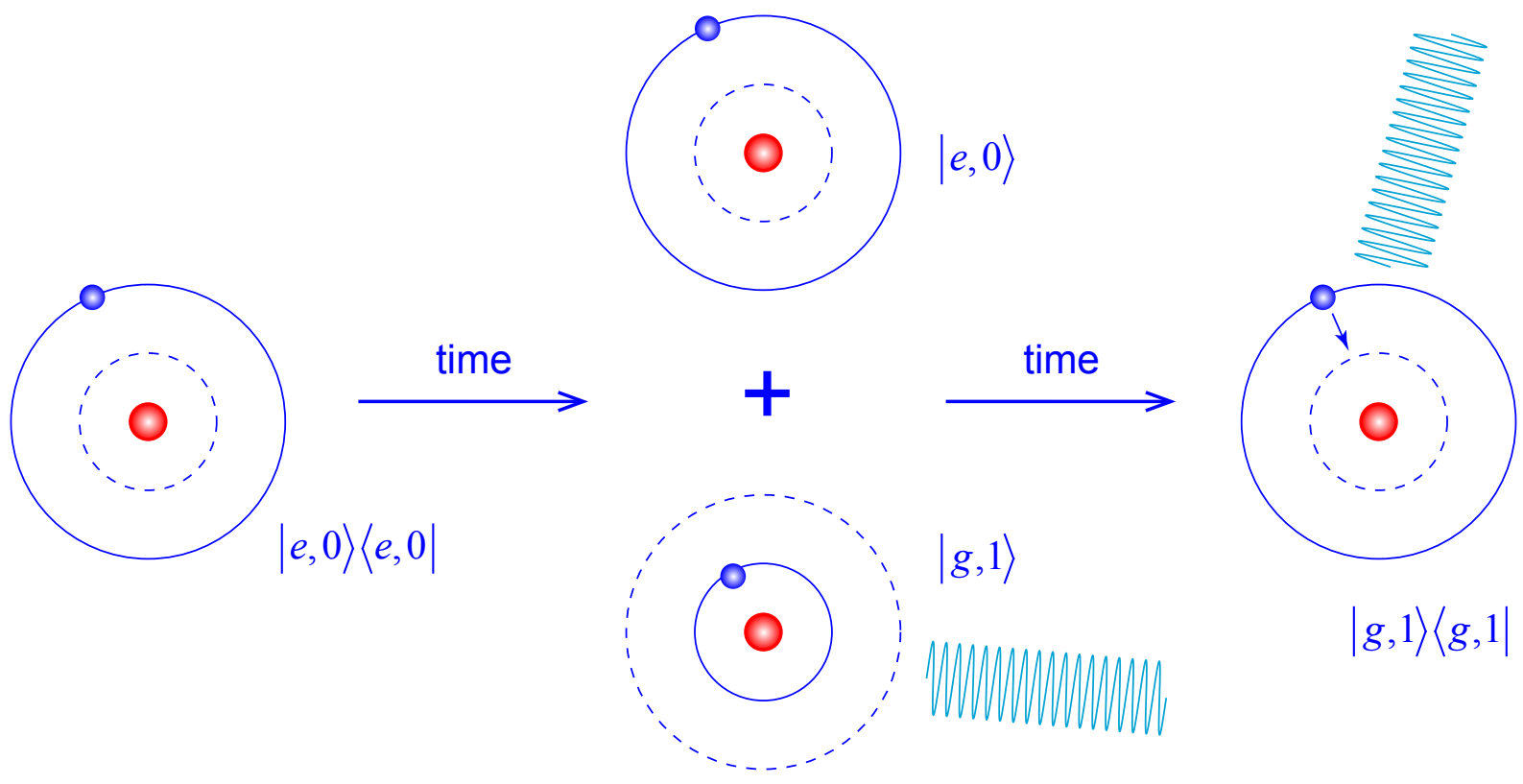

Figure 2: The decay of an excited atom (on the right) is a non-unitary process which breaks the superposition of states $|e, 0\rangle$ (excited atom, 0 photons) and $|g, 1\rangle$ (atom in ground state, 1 photons).

\section{Complex time}

We initially assume that $B$ is a single-particle state, postponing the discussion of the general case to a later stage. The basic idea is that the destruction of $B$ represents the stop of the time evolution of the wavefunction associated with the state $B$, and the creation of $B$ represents the start of the time evolution of the wavefunction associated with the state $B$. Time here refers to laboratory time, i.e. normal external time measured by an experimenter. If the particles created/destroyed are provided with a rest reference frame, this time corresponds (except for a Lorentz transformation) to each particle's proper time. In this case, it would be more appropriate to speak of stopping and starting in motion in their respective proper times.

The energy required to set in motion a body at rest, or to restore a body in motion to rest, is by definition kinetic energy. The kinetic energy of a particle in its rest reference is reduced to its rest energy, which is thus the energy required to set the particle in motion in its proper time (creation) or the energy released by the stop of such motion (annihilation); the involved process must therefore also define the mass of the particles created/destroyed. Therefore, proper time and mass both appear as emerging parameters in the description we are proposing.

The second feature of the model must be a proper characterization of the intermediate condition of timelessness, so to speak, between the destruction of $B$ and its successive re-creation. We follow the idea that the forerunner of time (its precursor) is a complex time $\tau=\tau^{\prime}+\imath \tau^{\prime \prime}$, in which $\tau^{\prime} \in\left[-\theta_{0},+\theta_{0}\right] \subset \mathbb{R}$ and $\tau^{\prime \prime} \in\left[0,+\theta_{0}\right] \subset \mathbb{R}$. The parameter $\theta_{0}$, which has the dimensions of a time interval, is assumed to be a new fundamental constant of Nature connected to the size of elementary particles, as detailed in a subsequent section.

We now focus on the creation of $B$. We assume the precursor of the outgoing wavefunction associated with state $B$ is as follows

$$
\Psi\left(y, \tau^{\prime}, \tau^{\prime \prime}\right)=Y(y) \Phi\left(\tau^{\prime}\right) \Lambda\left(\tau^{\prime \prime}\right)
$$

The factor $Y$ is only present if the particle created in state $B$ actually has spatial extension in the internal space-time coordinates $y$. This is the case of hadrons, although this factor is absent in the case of particles with no spatial extension, such as leptons. The other two factors are assumed to satisfy the following equations

$$
-\hbar^{2} \frac{\partial^{2}}{\left[\partial\left(2 \pi \tau^{\prime}\right)\right]^{2}} \Phi=\left(M_{s k} c^{2}\right)^{2} \Phi
$$

with the condition $\Phi=0$ for $\tau^{\prime} \leq-\theta_{0} / 2, \tau^{\prime} \geq+\theta_{0} / 2$ (the meaning of $M_{s k}$ is discussed below);

$$
-\imath \hbar \frac{\partial}{\left[\partial\left(\imath \tau^{\prime \prime}\right)\right]} \Lambda=\frac{\hbar}{2 \theta_{0}} \Lambda
$$

From Eq. 3, which is basically a Schrödinger equation in the imaginary component of complex time, it immediately follows that

$$
\Lambda \propto \exp \left(-\frac{\tau^{\prime \prime}}{2 \theta_{0}}\right)
$$


From Eq.2, which is the square of a Schrödinger equation in the real component of complex time, we get the even solutions

$$
\Phi \propto \cos \left[\left(n+\frac{1}{2}\right) \frac{2 \pi \tau^{\prime}}{\theta_{0}}\right]
$$

and the odd solutions

$$
\Phi \propto \sin \left(\frac{2 \pi n \tau^{\prime}}{\theta_{0}}\right)
$$

with integer $n \geq 0$. Given, in both cases, that

$$
n^{\prime}=n, n+\frac{1}{2}
$$

we have

$$
M_{s k} c^{2}=n^{\prime} \frac{\hbar}{\theta_{0}}
$$

We note that Eq. 4 can be rewritten in thermal form

$$
|\Lambda|^{2} \propto \exp \left(-\frac{E_{0}}{k T}\right)
$$

where $E_{0}=\hbar / \theta_{0}$ and the formal temperature

$$
T=\frac{\hbar}{k \tau^{\prime \prime}}
$$

has been introduced, which is infinite for $\tau^{\prime \prime}=0$, but assumes the minimum value for $\tau^{\prime \prime}=\theta_{0}$.

Finally, with regard to $Y(y)$, we note that when it exists, i.e. in the hadronic case, each single coordinate $y$ represents a distance in the internal space-time. If $\tau^{\prime}$ is interpreted as a kind of internal time of the particle, the chronological distance at which an internal observer places an internal event should be limited to the interval $\left[-\theta_{0} / 2,+\theta_{0} / 2\right]$, whichever observer is chosen. Therefore, the coordinate transformations that lead from one internal observer to another should retain the condition

$$
y \cdot y \leq\left(\frac{c \theta_{0}}{2}\right)^{2}
$$

which is precisely what defines a de Sitter space-time related to that single quantum jump. The space-time coordinates $y$ are, of course, internal coordinates that distinguish internal events not accessed by an outside observer. Hence there is an external Minkowskian relativity and an internal de Sitter relativity. $Y(y)$ presumably satisfies a wave equation which also includes terms related to the interaction between the subcomponents of the hadron. Leptons have no spatial extension and only the fluctuations in internal time $\tau^{\prime}$ described by Eqs. 5 - 8 exist for them.

\section{Physical meaning of complex time}

Before going further, it is worth pausing to consider the physical meaning of the real and imaginary parts of complex time $\tau$. Note that we are considering the timeless vacuum state following the destruction of $B$ and prior to its new creation: the system has stopped its course in external time and has not yet resumed it. Therefore, complex time $\tau$ is necessarily an internal time of this vacuum state, inaccessible to the external observer. From the perspective of external time, the timeless vacuum is an instant without duration; indeed, quantum jumps have no external duration.

It may be reasonably assumed that all particle states exist in potentia in this vacuum state as virtual fluctuations. However, the adjective virtual has to be intended in a radically different sense respect to the entirely fictitious virtual particles derived from the expansion of the $S$ operator; vacuum fluctuations are instead physically real and virtual is here used as a mere synonimous of unobservable. If a particle is at a chronological distance $\tau^{\prime \prime}$ from the singularity $\tau^{\prime \prime}=0$ (i.e. on the $\tau^{\prime \prime}$ ordinate axis in the complex time plane), this means that it is associated with an energy fluctuation with amplitude $\hbar / \tau^{\prime \prime}=k T$. Eq. 9 therefore provides the relative probability of such a fluctuation. The imaginary part $\tau^{\prime \prime}$ of complex time is therefore a measure of the energy amplitude of the fluctuation associated with that particle.

As seen previously, the real part $\tau^{\prime}$ is a kind of internal time of the vacuum, in which the wavefunctions of elementary particles live when they are dormant relative to external time (i.e. after their annihilation or before their creation). In this internal time, these functions are oscillatory, i.e. the vacuum is characterised by internal periodic phenomena; these are the different types of elementary particles that can be created or destroyed.

\section{Creation and annihilation of particles in a thermalized vacuum}

We now return to the discussion of the creation of state $B$. This is defined as a particular mapping of Eq. 1. This mapping firstly redefines the domain of the factors $\Phi$ and $\Lambda$, which now becomes the circumference, with centre $0+\imath 0$ and radius $\theta_{0}$

$$
\left(\tau^{\prime}\right)^{2}+\left(\tau^{\prime \prime}\right)^{2}=\left(\theta_{0}\right)^{2}
$$

The factor $\Phi$ becomes constant over the entire circumference in Eq. 12. The factor $\Lambda$ becomes

$$
\Lambda \propto \exp \left(-\frac{\tilde{\tau}}{2 \theta_{0}}\right)
$$


where

$$
\tilde{\tau}= \pm 2 n^{\prime} \iota \omega \quad \omega=\theta_{0} \arctan \left(\frac{\tau^{\prime \prime}}{\tau^{\prime}}\right)
$$

$\omega$ is the arc described on the circumference in Eq. 12 , counted in $\tilde{\tau}$ as positive if it is described in an anticlockwise direction and as negative if it is described in a clockwise direction. Eq. 13 can be written as

$$
\Lambda \propto \exp \left( \pm \imath M_{s k} c^{2} \frac{\omega}{\hbar}\right)
$$

which represents the phase factor in the particle's proper external time, if we identify this time with $\omega$. The appearance of the factor in Eq. 15 implies that $\Psi$ has resumed its course in laboratory time. If the + sign applies, we are looking at the creation of a particle (positive mass); if the - sign applies, this indicates the creation of an anti-particle (negative mass).

Eq. 15 is just the complex factor that expresses the rotation of an arc $\omega$ on the circumference in Eq. 12, travelled with the frequency $n^{\prime}$. Laboratory time is co-emergent with the de Broglie oscillation in Eq. 15 and with the particle's rest frame of reference. The magic of this triple emergence is not in the Wick rotation in Eq. 14 but in the transition from the complex plane to Eq. 12 . Once the domain of the factors $\Phi$ and $\Lambda$, initially two-dimensional, is reduced to a closed line, time is reduced to an onedimensional variable, whose domain can be traveled an infinite number of times. The infinite recurrences of a given domain point constitute the external time line.

Ultimately, the mapping converts a rectangular domain of complex time $\tau^{\prime}+\imath \tau^{\prime \prime}$ into a circular domain $\omega$ (Fig. 3). The fluctuations of amplitude $\tau^{\prime \prime}$ become an oscillation $\Phi \Lambda$ of fixed amplitude, represented by a vector with a free end on the circumference in Eq. 12 and the point of application in $0+t 0$. The frequency of the previous oscillation $\Phi$ in $\tau^{\prime}$ becomes the frequency of $\Phi \Lambda$ in $\omega$. Thus, there is a recoding of the relevant information and the transition from a fluctuation to a state vector of constant norm persistent in $\omega$.

We now come to the mapping action on the coordinates $y$ and on the factor $Y(y)$. Firstly, the new quantum of the particle's internal time is $\hbar / M_{s k} c^{2}=\theta_{0} / n^{\prime}$ since, based on the reasoning presented above, the internal chronotope (if any) should change into a de Sitter chronotope with radius $c \theta_{0} / n^{\prime}$. We refrain from further discussion of the implications for the structure of hadrons, leaving this for future research. We limit ourselves to observing that the coordinates $y$ and the factor $Y(y)$ have to undergo consequent scale transformations.

However, the appearance of proper time leads to the appearance of external spatial coordinates. Indeed, a generic observer in motion respect to the particle sees the particle's proper time line as its trajectory in space.
Relativistic covariance therefore requires the appearance of a complete system of external space-time coordinates $x$ and the simultaneous appearance of a factor $X(x)$ in the wavefunction of the particle emerging from the vertex. The outgoing wavefunction is therefore, in conclusion (and omitting internal dynamics in the case of hadrons), $\Psi=X \Lambda$. We have $X(x)=\langle x \mid B\rangle=\langle B \mid x\rangle^{*}$, i.e., the outgoing wavefunction is the complex conjugate of the incoming one. In general, $X$ will be a spinor, thus the conjugation includes a transposition. This spinor represents the new initial condition for the unitary evolution described by the appropriate wave equation (Dirac, Proca, etc.) possibly with external fields. Each component of $X$ satisfies the Klein-Gordon equation, which can be written in the usual form

$$
-\hbar^{2} D^{2} X=\left(M_{s k} c\right)^{2} X
$$

where $D^{2}$ is the usual D'Alembert operator in Minkowski coordinates $x$. Note that the outgoing $X(x)$ is generally not an eigenstate of the position, and so the outgoing particle is normally delocalized.

The destruction of the state $B$ entering the vertex is described by the exact opposite mapping. The constraint by Eq. 12 is removed and we return to the complex time plane. The factors $\Phi$ and $\Lambda$ resume their original shape. External time disappears, and with it the external spatial coordinates, too. There is no longer a rest frame of reference for the particle, or a de Broglie oscillation. The archaic vacuum state prior to any physical manifestation is restored.

We are now able to address the general case involving a plurality of particles entering and leaving the vertex. The crucial observation is that the timeless vacuum state is described by two temporal parameters but no spatial parameter. In other words, dormant state precursor of $B$ (after $B$ annihilation and before its re-creation) does not contain external space-time coordinates $x$; these are created or destroyed on the occasion. The quantum jump is therefore an aspatial event, and this allows us to define $B$ as an appropriate entanglement of the amplitudes of the individual particles, respectively entering or exiting, i.e. linear combinations of products of these amplitudes. The amplitude of each individual particle is defined on the specific configuration space of that particle, thus $B$ will live in the total configuration space of all the particles involved. It is also possible to represent $B$ through second quantization creation/annihilation operators defined on a suitable Fock space, and this leads to the quantum field theory description.

The following is an indicative example. Let $A$ be the product of the two (non entangled) spatial wavefunctions of two identical particles of spin $\frac{1}{2}$ and their spin wavefunction, which we assume to be a singlet. Let $B$ be the 


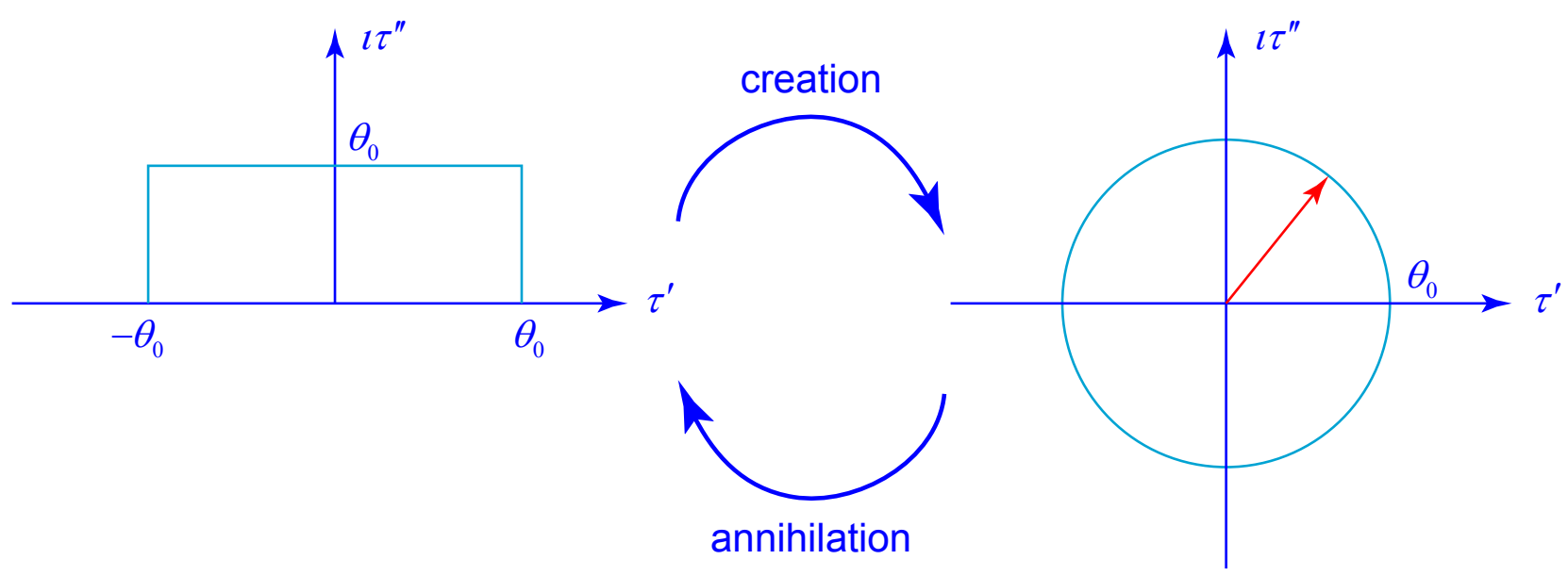

Figure 3: Illustration of the wavefunction creation and annihilation in the proposed model.

product of the spatial wavefunctions of the same two particles (the one peaked around the position $x_{0}$ at time $t_{0}$, the other identical to that in $A$ ) and their spin wavefunction equivalent to one of the two components of singlet $A$, considered along the $z$ axis. Let us consider the preparation $|A\rangle\langle A|$ followed by detection $|B\rangle\langle B|$. This second event refers to the interaction, at time $t_{0}$, between one of the two particles and a measurement device placed in $x_{0}$, with simultaneous measurement of its spin along the $z$ axis. In the language of present model the aspatial (and therefore in a way ubiquitous) background has accepted $B$ in input and returned it as a new initial state, thus inducing the spatial localization of one of the particles and a sharper definition of spin of both particles. We consider here that the projectors $|A\rangle\langle A|| B\rangle,\langle B| \ldots$ are acting on their right. If we consider them acting on the left we have the description of the same phenomenon but reversed in time. This example illustrates the connection between background aspatiality and non-separability of an entangled state. This second form of nonlocality follows from the first, which is in this sense more radical.

\section{Digression on skeleton mass and self-interaction}

The interpretation of the mass $M_{s k}$ is important. The creation (annihilation) of particles always occurs in a vertex of interaction with other particles and has a finite duration, so the mass of each particle becomes indeterminate by virtue of Heisenberg relationships. Only the free particle, i.e. the asymptotic particle state exiting from the vertex or entering it, has a definite mass. In terms of normal quantum field theory language, the particle is born bare and is then dressed by its own self-interaction processes, so that the nascent (bare) particle is not the particle physically observed away from the vertex (dressed particle).

We can assume the physical mass $m$ of the particle to be the sum of the nascent mass $M_{s k}$ and a term $\varepsilon / c^{2}$ associated with the self-interaction. If energy $m c^{2}$ is applied to the vacuum, the creation of that particle in it becomes possible, i.e. its actual emergence from the vertex. From this perspective, the time interval $\theta_{0} / n^{\prime}$ represents a sort of minimum chronological distance between two virtual self-interactions belonging to the same interaction vertex. The creation sequence of the mass is therefore as follows:

(1) The particle is initially massless (vacuum state);

(2) Its localization in an interaction event, for a duration of $\theta_{0} / n^{\prime}$, requires an amount of energy equal to the ratio of $\hbar$ and this duration; this ratio is the rest energy in Eq. 8 . Thus the particle skeleton mass $M_{s k}$ appears.

(3) The particle self-interacts for a duration of $\hbar / M_{s k} c^{2}$, and therefore on a scale of lengths equal to $\hbar / M_{s k} c$. The total mass $m$ is therefore the sum of the skeleton mass and the $\varepsilon / c^{2}$ mass derived from this self-interaction.

(4) There is no self-interaction for chronological distances from the vertex greater than $\hbar / m c^{2}$; the particle's rest energy is the minimum energy required to extract the particle from the vertex.

(5) The actual Klein-Gordon equation does not contain the skeleton mass $M_{s k}$, but the actual mass $m$. In other words, a term $\varepsilon / c^{2}$ must be added to the right-hand side of Eq. 16 .

(6) If we interpret the skeleton mass $M_{s k}$ as bare mass, we obtain the interesting result that it is finite.

If $n^{\prime}=0$, only the term of self-interaction survives in $m$. Only a fraction of the energy $\hbar / \theta_{0}$ needed to locate the particle in a temporal extension $\theta_{0}$ is used, expressed by the dimensionless self-coupling constant $\left(g^{2} / \hbar c\right)$. This energy is therefore $g^{2} /\left(c \theta_{0}\right)$, and the particle is delocalized to a dressing region $\left(\hbar c / g^{2}\right)$ times larger than $c \theta_{0}$. It is possible that this is the situation of lighter particles, 
i.e. electrons and neutrino mass eigenstates. With electrons, the self-interaction will be essentially electrostatic and therefore the self-interaction energy will be $e^{2} /\left(c \theta_{0}\right)$, where $e$ is the elementary electric charge. Equating this expression to the rest energy of the electron, we obtain $c \theta_{0}$ is the classical radius of the electron. As a result, the fundamental skeleton mass interval $\hbar / \theta_{0}$ seen in Eq. 8 is approximatively $70 \mathrm{MeV}$.

Although a serious formulation of the entire conjecture would require further explanation of the relationship between $n^{\prime}$ and the internal quantum numbers (and a precise calculation method for the self-interaction term), it should nevertheless be noted that the bare mass in this context is finite and that there are no divergences.

In our model, $\theta_{0}$ coincides, at least by a factor of $\frac{2}{3}$, with the chronon introduced by Caldirola in his classical model of the electron [39,40]. Note, however, that the fundamental interval $\theta_{0}$ is a property of the vacuum or background that manifests itself in external time only as a minimum duration $\theta_{0} / n^{\prime}$ associated with the localization of the particle in a quantum jump. This interval does not play any role in the next (or previous) unitary evolution of the particle state vector, which is described by current quantum formalism. However, it could be relevant in selecting base states (elementary particles) and defining their properties, such as a finite mass spectrum. A more formal illustration of the scheme can be the following. Let us consider a massive particle endowed with a proper rest frame of reference. Let $t$ be the particle proper time and $\sigma$ a scalar quantity (respect to the Poincaré group of coordinate transformations) such that $t=t(\sigma)$ and

$$
\sigma_{2}-\sigma_{1}=\left|t\left(\sigma_{2}\right)-t\left(\sigma_{1}\right)\right|
$$

The integrals of $d \sigma$ and $d t$ along a given segment of a four-dimensional line respectively measure its length and the extension in the particle proper time $t$. They coincide if the time orientation of the line is the same in each point of the segment. From this definition, the following relations can be immediately derived

$$
\frac{d t}{d \sigma}=\lim _{\sigma_{2} \rightarrow \sigma_{1}} \frac{t\left(\sigma_{2}\right)-t\left(\sigma_{1}\right)}{\sigma_{2}-\sigma_{1}}= \begin{cases}+1 & \text { for } t\left(\sigma_{2}\right)>t\left(\sigma_{1}\right) \\ -1 & \text { for } t\left(\sigma_{2}\right)<t\left(\sigma_{1}\right)\end{cases}
$$

\section{(18)}

These two relations can be summarized in a single equation

$$
\frac{d t}{d \sigma}=\gamma_{0}=\left(\begin{array}{cccc}
1 & 0 & 0 & 0 \\
0 & 1 & 0 & 0 \\
0 & 0 & -1 & 0 \\
0 & 0 & 0 & -1
\end{array}\right)
$$

The left hand derivative is, in this case, an operator with eigenvectors

$$
\left(\begin{array}{l}
1 \\
0 \\
0 \\
0
\end{array}\right),\left(\begin{array}{l}
0 \\
1 \\
0 \\
0
\end{array}\right),\left(\begin{array}{l}
0 \\
0 \\
1 \\
0
\end{array}\right),\left(\begin{array}{l}
0 \\
0 \\
0 \\
1
\end{array}\right)
$$

having +1 and -1 as respective eigenvalues. By setting $x_{0}=c t$ we therefore have

$$
\frac{d x_{0}}{d \sigma}=c \gamma_{0}
$$

In a frame of reference in uniform rectilinear motion with respect to the particle rest frame, this relationship takes the following form

$$
\frac{d x_{\mu}}{d \sigma}=c \gamma_{\mu} ; \mu=0,1,2,3
$$

All the spacetime coordinates in this relationship have an implicit dependency on $\sigma$. If the particle wavefunction $\psi$ does not explicitly depend on $\sigma$, but only through the coordinates, we have

$$
\frac{1}{c} d_{\sigma} \psi=\gamma^{\mu} \partial_{\mu} \psi
$$

In general, the gamma operators will be the Dirac matrices and the wavefunction will therefore be a spinor. Given the assumptions made in this section, the outgoing wavefunction from the interaction vertex is

$$
\psi=\phi\left(x_{\mu}\right) \exp \left(-\imath M_{s k} c^{2} \sigma / \hbar-\imath c^{2} \sigma \delta M / \hbar\right)
$$

This is the result of mapping, with the addition of a corrective term to the exponent containing the perturbative correction $\delta M=\varepsilon / c^{2}$ to the skeleton mass due to the particle self-interaction (limited to the vertex where it is created). To free the particle and remove it from the vertex it is necessary to administer a $M c^{2}$ energy, with $M=M_{s k}+\delta M$. The ordinary Dirac equation for a free particle of mass $M$ thus follows

$$
\imath \hbar \gamma^{\mu} \partial_{\mu} \phi=M c \phi
$$

It is possible to treat the particle subjected to gauge fields through the typical replacement of four-momentum with the canonical four-momentum. If this procedure is applied at the interaction vertex where the particle is created, taking into account only the particle self-field, it produces expressions such as the following (where we consider only the electromagnetic self-interaction of a particle with charge $e$ and self-field $A_{\mu}$ )

$$
\delta M=-\frac{e}{c^{2}} \int \bar{\phi} \gamma^{\mu} A_{\mu} \phi d V
$$


The integral is extended to a volume of diameter $\hbar / M_{s k} c$ around the vertex (we assume that $M_{s k} \neq 0$ ) and the minimum interaction distance is $c \theta_{0} / n^{\prime}$, which is equal to this diameter. In the particle rest frame of reference this is a Coulomb integral whose order of magnitude is

$$
\approx \frac{e^{2}}{\left(\frac{\hbar}{M_{s k}}\right)}=\left(\frac{e^{2}}{\hbar c}\right) M_{s k} c^{2}=\alpha M_{s k} c^{2}
$$

where $\alpha$ is the fine structure constant.

A separate case, discussed above, is the electron for which $M_{s k}=0, M=\delta M$ and the integral in Eq. 26 does not exist. In the external time of the observer the electron is localized in the interval $\hbar / M c^{2}$, while in the internal time $\tau^{\prime}$ it is a virtual oscillation with zero frequency and duration $\theta_{0}$. The ratio between the external and internal temporal extension represents the number of times in which the interval $\hbar / M c^{2}$, containing a single real electron, contains the electron as virtual oscillation. The inverse of this number is the electron adimensional constant of interaction, that is, the probability to actualize a virtual electron in response to an interaction. It is essentially the electromagnetic fine structure constant (the electron interacts substantially through the electromagnetic field). We therefore have $\hbar / M c^{2} \theta_{0}=\hbar c / e^{2}$, from which we obtain $\theta_{0}=e^{2} / M c^{3}$ namely $\hbar / \theta_{0}=70 \mathrm{MeV}$. We could describe the situation by saying that the coupling with a real photon consists of the actualization of one (on average) of $\hbar c / e^{2}$ virtual electrons. In the external time period $T_{e}=h / M c^{2}$ the phase of the electron in Eq. 24 varies by $2 \pi$. Since the electron spin is $\frac{1}{2}$, we would obtain the same phase variation by rotating the electron around any spatial axis of a $4 \pi$ angle. In this sense the phase pulsation $2 \pi / T_{e}$ is equivalent to a spatial rotation pulsation $4 \pi / T_{e}=2 M c^{2} / \hbar[41-43]$. It is possible to define the electric current $I=e\left(2 M c^{2} / \hbar\right)$ associated with this rotation and the rotation radius $L=c\left(\hbar / 2 M c^{2}\right)$. Therefore, the magnetic moment is defined

$$
\frac{1}{c} I L^{2}=\frac{e \hbar}{2 M c}
$$

which is the Dirac magnetic moment of the electron. On the other hand, in an interaction vertex the not yet actualized electron appears as a virtual fluctuation of minimum temporal extension $\theta_{0}$, therefore associated with a transitory phase pulsation $2 \pi / \theta_{0}$ corresponding, for the same principle, to an angular pulsation $4 \pi / \theta_{0}$. By setting $I=e\left(4 \pi / \theta_{0}\right), L=c\left(\theta_{0} / 4 \pi\right)$, and taking into account that $c \theta_{0}=\alpha \hbar / M c$, we achieve an additional magnetic moment from this high frequency oscillation equal to

$$
\frac{1}{c} I L^{2}=\frac{e \hbar}{2 M c} \frac{\alpha}{2 \pi}
$$

which is the anomalous magnetic moment at the first order. The magnetic moment is naturally a latent property which becomes effective only in presence of an external magnetic field that, with its direction, selects a spatial rotation axis.

However, a transitory state as that of a virtual electron in an interacion vertex cannot be described only by the monochromatic pulsation $2 \pi / \theta_{0}$ and a wave packet with pulsations ranging from $2 \pi / T_{e}$ to $2 \pi / \theta_{0}$ will be actually involved. The Fourier components will exchange virtual photons so generating radiative corrections of higher order to the magnetic moment. Under this perspective $\alpha$ represents the probability of actualization of a virtual selfinteracting electron, what justifies the perturbative origin of $M$.

In summary, the introduction of the chronon as a vacuum constant seems to enable, at least in principle, the derivation of a finite mass spectrum for elementary particles. The finite value of the chronon implies a finite value of the skeleton mass, which appears in the phase factor in Eq. 24 in a completely adynamic manner, together with the particle (external) proper time. The interval of proper time given by the inverse (in natural units) of the mass skeleton represents the duration of the particle self-interaction and also the minimum interval between two virtual self-interactions. The contribution of self-interaction to the effective mass of the particle is therefore finite. The finite value of the effective mass in turn justifies the finite value of the magnetic moment.

The conjugation of the adynamic mechanism for generation of the masses (such as mapping) with quantum field theory requires further elaboration and here we only highlight the effects that the chronon has on the choice of the cut-off. For example, consider the following relationship for the mass $M$ of a fermionic $\frac{1}{2}$ spin field coupled with a gauge field of spin 1 and (non null) mass $\mu$, with an adimensional coupling constant $g$ [44]

$$
M=M_{0}+\frac{4 g^{2} M}{2 \pi^{2} \mu^{2}}\left[\Lambda^{2}-M^{2} \log \left(\frac{\Lambda}{M}\right)^{2}\right]
$$

where $M_{0}$ is the fermion bare mass and $\Lambda$ is the cutoff. Setting $M_{0}=M_{s k}$ and $\Lambda=M_{s k}$ we achieve a transcendental equation in $M$ which can be solved iteratively. For example for $2(g / \pi \mu)^{2}=0.1, M_{s k}=1$ we get $M=1.16459 \ldots$ a clearly finite result. The skeleton mass is defined by the minimum time extension of the particle and, therefore, constitutes a cut-off for virtual coupling; this fact leads to a finite mass. A more consistent treatment, however, should lead to a unification of the ideas expressed in this paper with the quantum field theory formalism, a task which we must leave for a future work. 


\section{Suggestions for hadronic physics}

The equation

$$
|\Lambda|^{2}=\exp \left(-\frac{\tau^{\prime \prime}}{\theta_{0}}\right)
$$

represents the background as a set of thermostats with different absolute temperatures, which are included in the range between $T=\hbar / k \theta_{0}$ (for $\tau^{\prime \prime}=\theta_{0}$ ) and $T=\infty$ (for $\tau^{\prime \prime}=0$ ). The thermostat corresponding to the temperature $T$ shall contribute to the creation/annihilation of a particle with rest energy $M c^{2}$ at that temperature (at $\tau^{\prime \prime}=\hbar / k T$ ) through the heat exchange

$$
d Q=M c^{2} \frac{\exp \left(-\frac{\tau^{\prime \prime}}{\theta_{0}}\right) d \tau^{\prime \prime}}{\int_{0}^{\theta_{0}} \exp \left(-\frac{\tau^{\prime \prime}}{\theta_{0}}\right) d \tau^{\prime \prime}}
$$

equal to the product of the rest energy by the probability of its release. Now imagine an equivalent thermostat such that: 1) the entropy variation of the whole set of thermostats is equal in value to the entropy variation of the equivalent thermostat; 2 ) the sum of the thermal contributions of the different thermostats is equal to the total thermal contribution of the equivalent thermostat. Having to do with reversible processes only, these two conditions are reflected in the relation

$$
\int_{\tau^{\prime \prime}=0}^{\tau^{\prime \prime}=\theta_{0}} d Q / T=\frac{\int_{\tau^{\prime \prime}=0}^{\tau^{\prime \prime}=\theta_{0}} d Q}{T_{H}}
$$

where $d Q$ is the heat exchanged by the thermostat at temperature $T$ and $T_{H}$ is the temperature of the equivalent thermostat. Now add the additional condition that the thermal exchanges $d Q$ occur in the form of rest energy of massive particles exchanged within the same interaction vertex, i.e., in a contact interaction. Thus, we basically limit ourselves to the strong interaction between hadrons entering a vertex where a quark exchange occurs, with the possible creation/annihilation of quark pairs. The exchange takes place within that same vertex, thus generating new hadrons exiting from it. Conversely, we exclude electroweak and gravitational interactions from our consideration because the absorption and emission of their gauge quanta occur in distinct vertices; furthermore, photon and graviton are massless.

Substituting Eq. 32. into Eq. 33 we obtain

$$
k T_{H}=\frac{\hbar}{\left\langle\tau^{\prime \prime}\right\rangle}=\frac{\hbar}{\theta_{0}} \frac{e-1}{e-2}
$$

where

$$
\left\langle\tau^{\prime \prime}\right\rangle=\frac{\int_{0}^{\theta_{0}} \tau^{\prime \prime} \exp \left(-\frac{\tau^{\prime \prime}}{\theta_{0}}\right) d \tau^{\prime \prime}}{\int_{0}^{\theta_{0}} \exp \left(-\frac{\tau^{\prime \prime}}{\theta_{0}}\right) d \tau^{\prime \prime}}=\theta_{0} \frac{e-2}{e-1}
$$

If, according to the argument developed in the previous section, we assume $\hbar / \theta_{0}=70 \mathrm{MeV}$ we obtain $k T_{H}=$ 167.5 MeV. This value is practically coincident with that currently accepted for the Hagedorn temperature (which is included in the range $160-190 \mathrm{MeV}$ ). In order to exclude a mere numerical coincidence, we now consider the case where the equivalent thermostat may be placed in thermal contact with a process consisting of the creation of a single hadron of rest energy $M c^{2}$ at temperature $T$ (at $\tau^{\prime \prime}=\hbar / k T$ ). The related (finite) heat transfer is $Q_{0}=M c^{2}$. The system made of equivalent thermostat and the process undergoes a total entropy variation equal to the difference between Eq. 33 and the entropy variation associated with the process

$$
\Delta S=-\frac{Q_{0}}{k T}+\frac{\int_{\tau^{\prime \prime}=0}^{\tau^{\prime \prime}=\theta_{0}} d Q}{k T_{H}}=-\frac{M c^{2}}{k T}+\frac{M c^{2}}{k T_{H}}
$$

The probability of the fluctuation corresponding to the actualization of the hadron is therefore proportional to

$$
\exp (\Delta S)=\exp \left(-\frac{M c^{2}}{k T}+\frac{M c^{2}}{k T_{H}}\right)
$$

The number of fluctuations likely to occur within the localization volume $(\hbar / M c)^{3}$ of the exchanged hadron can be estimated from their thermal volume $(\hbar c / k T)^{3}$ as

$$
\approx\left(\frac{\hbar}{M c}\right)^{3}\left(\frac{\hbar c}{k T}\right)^{-3}=\left(\frac{k T}{M c^{2}}\right)^{3}
$$

The partition of the system made of equivalent thermostat and the process will therefore be the integral in $M$ of the following expression

$$
\left(\frac{k T}{M c^{2}}\right)^{3} \exp \left(-\frac{M c^{2}}{k T}+\frac{M c^{2}}{k T_{H}}\right)
$$

It involves the same density of hadron mass states

$$
\rho(M) \propto M^{-3} \exp \left(\frac{M c^{2}}{k T_{H}}\right)
$$

as that derived from Hagedorn's [45] original statistical bootstrap model; this confirms the identification of $T_{H}$ with the Hagedorn temperature. To conclude, the thermostat equivalent to the background is manifested in each vertex of strong interaction between hadrons, and it consists of the self-similar pattern of fluctuations of quark and gluon plasma, according to the current interpretation of Hagedorn's model [46, 47]. This pattern will absorb heat at $T>T_{H}$ (released by the annihilation of hadrons entering the vertex) and will release it at $T<T_{H}$ through the creation of new hadrons, thus originating a temperature $T_{H}$ characteristic of the hadronization process. Hadrons 
entering the vertex will be annihilated at the same temperature, at which the deconfinement of quarks and gluons will occur (Fig. 4). We believe that the salient point here consists of the relation between the Hagedorn temperature, which expresses the time-scale in Eq. 35 at which the scale invariance of the fluctuations is broken, and the constant $\theta_{0}$. The value of this constant, as derived from a discussion about the electron, produces the correct value of $T_{H}$. This connection between the lepton and hadron worlds, resulting from the universality of the constant $\theta_{0}$, remains hidden in the conventional treatment.

The introduction of the fundamental constant of nature $\theta_{0}$ also has another effect. It implies the existence of a fundamental moment of inertia $\hbar \theta_{0}$, which should not be interpreted in the classical terms of a mass distribution, but rather as a conversion factor between time intervals characteristic of elementary particles and their angular momentum. Before re-scaling $\theta_{0} \rightarrow \theta_{0} / n$ of de Sitter time of the particle micro-universe, the relevant moment of inertia is $m c^{2} \theta_{0}^{2}=n \hbar \theta_{0}$, where $m$ is the particle mass (substantially, the skeleton mass) and $n$ is the ratio of the skeleton mass and $70 \mathrm{MeV}$; it probably plays a role in defining the Regge trajectories [48]. After re-scaling, the relevant moment of inertia is instead

$$
I=m c^{2}\left(\frac{\theta_{0}}{n}\right)^{2}=\left(n \frac{\hbar}{\theta_{0}}\right)\left(\frac{\theta_{0}}{n}\right)^{2}=\frac{\hbar \theta_{0}}{n}
$$

The angular momentum $J$ of the particle is given by the product of $I$ for an angular frequency $\omega$ typical of the particle. It is natural to set $\omega=j /\left(\theta_{0} / n\right)$, where $j$ is the eigenvalue of the spin (even in the dormant state, the wavefunction of a particle nevertheless has a total spin eigenvalue of $j$, which defines its number of components). Thus

$$
J=I \omega=\left(\frac{\hbar \theta_{0}}{n}\right)\left(\frac{n j}{\theta_{0}}\right)=j \hbar
$$

Eq. 42 holds for all particles, yet for hadrons a word of caution seems necessary. Indeed, the quark substructure could admit a different time-scale $\theta^{\prime}$ from de Sitter time $\theta_{0} / n$ of the hadron it belongs to. In this case, the angular momentum associated with this substructure is therefore

$$
J^{\prime}=I \omega^{\prime}=\frac{\hbar \theta_{0}}{n} \frac{j}{\theta^{\prime}}
$$

An external probe capable of selectively detecting this substructure will therefore not see the kinematic spin $J$, but rather the apparent spin

$$
J^{\prime}=J \frac{\theta_{0}}{n \theta^{\prime}}
$$

If the hadron is a polarized proton, the probe can also be a polarized charged lepton (electron or muon). In a process of deep inelastic scattering of the probe on the proton, characterized by a square of the transferred fourmomentum $Q^{2}$ and a fraction $x$ of the hadron momentum carried by the interacting quark, the spin seen by the probe is given by the integral of the quark spin structure function $g_{1}(x)$

$$
\frac{1}{2} \int_{0}^{1} g_{1}(x) d x=\frac{\Delta \Sigma}{2}
$$

The experiments carried out by several collaborations reveal that $\Delta \Sigma \approx 0.25$ instead of 1 as expected from the quark model [49,50]. From our point of view, however, the function $g_{1}(x)$ should be scaled by the factor $n \theta^{\prime} / \theta_{0}$ independent from $x$. Since $n=13.4$ for the proton, this rescaling provides $\Delta \Sigma=1$ if $\theta^{\prime} \approx \theta_{0} / 3$. According to this point of view, the eigenvalue $j$ of the proton spin is always derived from the contributions of individual quarks according to the rules of the quark model, but the scattering process measures $J^{\prime}$ instead of the correct kinematic spin $J$.

The reason for the result $\theta^{\prime} \approx \theta_{0} / 3$ has yet to be determined. Since each quark is in one of three possible, and statistically equivalent, colour states, we can conjecture that the interval $\theta^{\prime}$ derives from the ratio between the fundamental interval $\theta_{0}$ and the number of these states. If so, the introduction of the constant $\theta_{0}$ would provide a different way of looking at the proton spin crisis and the contribution of the sea of virtual quarks and gluons.

\section{Comparison with other models}

In this paper we have repeatedly stressed that the state vector reduction postulate (von Neumann's projection postulate) is the real expression of the quantum discontinuity. This discontinuity finds its complete realisation in quantum jumps that occur in quantum systems as a result of interactions with internal degrees of freedom or external systems. Among the latter, we must include coupling with measuring devices that represent only one possibility among many. Otherwise it would be difficult to understand how gaseous oxygen and hydrogen combine giving molecules perfectly defined as water, when placed inside a container with entirely opaque walls and without any observation of the process. The scope of the von Neumann postulate then goes well beyond the measurement procedures; it is rather an essential ingredient for formulating the quantum mechanics.

Von Neumann's postulate, on the other hand, does not specify the ontology for the reduction process; this specification must, in any case, be compatible with the process of unitary evolution of the state vector between two successive collapses, described by the quantum mechanical equations of motion. Otherwise, we would be dealing 


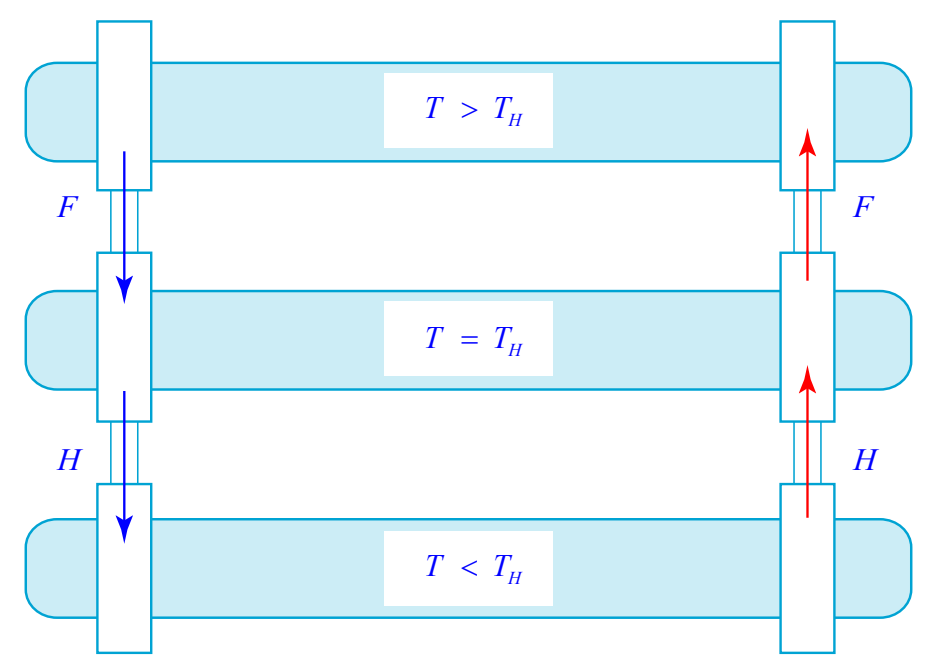

Figure 4: The Hagedorn thermostat. $F=$ self-similar pattern of virtual fluctuations; $H=$ hadrons; $F \rightarrow H$ heat flow $=$ hadronization; $H \rightarrow F$ heat flow = deconfinement.

with a theory that is entirely different from quantum mechanics and this is what occurs with the dynamic reduction mechanisms invoked by Ghirardi-Rimini-Weber [5] or by Penrose [51]. The minimal ontology of the projection operator proposed in this paper is fully consistent with usual quantum mechanics. It does not lead to new effects on the dynamics, but possibly only to constraints on the selection of the states (for example, particle masses). The possibility has been considered that the unitary evolution process of the state vector is, under appropriate conditions, sufficient to reduce the wavefunction. Within the area of quantum measurement theory, this possibility has already been explored in early models of Milan-Pavia [2] and Rome schools [52] and has re-emerged with the principle of decoherent histories. Although this approach is valid in the field of quantum measurement, it seems, however, to have less effect on interaction micro-events between micro-entities. The idea of the decoherent histories, proposed in particular by Zeh [53] and Zurek [54], requires the interaction of micro-entities with an environment with many degrees of freedom. Average operations on environmental degrees of freedom lead to mixtures of states of the system composed of micro-entities and apparatus, which are effectively indistinguishable from those derived as a result of the action of projection operators defined on a base selected by dynamic itself. From our point of view, the measurement processes are expressions of quantum discontinuity like the atomic quantum jumps which can occur in fully decoupled and virtually isolated quantum systems. Consider, for example, the electromagnetic emission from neutral galactic hydrogen which can be measured by any radio-telescope operating at a wavelength of $21.1 \mathrm{~cm}$. This emission is generated by the transition between the two levels of the hyperfine structure of neutral hydrogen atoms that are virtually isolated in interstellar space (their numerical density is often lower than or approximately equal to 1 $\mathrm{cm}^{-3}$ ). Quantum state reduction therefore occurs also in entirely decoupled systems and cannot be caused by the interaction with the surrounding environment. In this example, decoherence should in addition act on an absolutely improbable entanglement of emitting atoms with the receiving radio-telescope, two physical systems that have never been in contact. On the other hand, if the reduction process is assumed already at the atomic or molecular level in the form of quantum jumps, the measurement process can easily be interpreted as a quantum jump of the system composed of measurement apparatus and micro-entities. By doing so, there is no need to hypothesize the splitting of the state vector of this system in a multiplicity of independent worlds, as assumed by Everett. Moreover, it seems appropriate to mention the similarities and differences between our approach and the two-state formalism proposed by Aharanov, Vaidman and collaborators [55, 56]. In both cases, time symmetry is emphasized and the propagation of backward state vector is acknowledged on an equal footing with the forward vector. In our view, the main difference is the fact that the one, proposed by Aharanov and colleagues, is a formalism and not an ontology. This formalism assumes pre-selection and post-selection, followed by the application of the Aharanov-Bergmann-Lebowitz rule (in the original version or one of its variants) for calculating the relevant averages. It thus implicitly assumes that it is possible to perform an initial preparation of the system and a complete measurement of it. The real issue, however, is exactly how to ensure these conditions necessary for the applicability of the formalism which is, in itself, correct. 
It is not a coincidence that the two main supporters of this idea have identified this logical gap and have responded in their own way, opting for different ontologies [57]. Vaidman opts for a time-reversal version of Everett manyworlds model; Aharanov states he is not ready for that step to which he would (reluctantly) prefer an objective collapse. He assumes, in each case, the physical reality of the backward vector. This option inevitably leads to the transaction concept and its importance in the context of the two-state formalism is also considered by Elitzur and Cohen [58].

\section{Conclusions}

We proposed an extremely preliminary model of what a quantum jump is, in the broad sense of a discontinuous change in the quantum state of a system eventually related with particle localization in time. Our aim was to focus on the urgent need to provide the concept proposed by Bohr a century ago in the context of atomic theory with formal maturity, as this is the only concept from his model that is still not fully formalized. The matter was reopened by Erwin Schrödinger in an article written in 1952, where he states

It is better to consider a particle not so much as a permanent entity, but rather as an instantaneous event (...). At times these events form chains that give the illusion of something permanent [59].

At the core of the proposed model is the concept of the circularity of the time coordinate that appears in the wavefunction of a particle, a circularity expressed by the de Broglie oscillation. The annihilation of the wavefunction is described through the removal of the topological constraint of circularity. The reverse process describes its creation. The removal of the circularity constraint leads to a vacuum state represented by a complex time that is without temporality, in the ordinary sense of the term, and spatiality. Oscillations occur in the real part of complex time that represents the internal state of the various elementary particles contained in the vacuum and which can be extracted from it or lead back to it. These particles are present in the form of virtual fluctuations and the imaginary part of complex time describes the energy amplitude of these fluctuations. This vacuum structure seems to introduce new constraints to the structure of elementary particles and their internal dynamics, resulting from the introduction of a new constant of nature with the dimensions of a time interval, which we assume to be identifiable with Caldirola's chronon. Numerically, the chronon is the time taken by light to travel a distance equal to the classical radius of the electron. The constraints in question are perhaps reflected in already known facts, such as the proton spin crisis or the Hagedorn thermostat.

We have tried to show how the hypothesis of a time precursor allows to introduce nonlocality $a b$ initio, in a consistent way with both the non-separability of entangled states and the quantum equations of motion as that of Dirac. It is possible therefore, in principle, to find a connection with the more usual, stochastic hydrodynamic representation of these equations and the role there played by the Bohm potential [60]. It will not be specified enough that nonlocality and entanglement are two distinct phenomena, although intimately linked to the comlex background of quantum systems. This fact has been well pointed out by some recent works in quantum information [61-63]. On the other hand, we have discussed the relations with the transactional representation and the two states formalism. In our opinion, the quantum mechanics is not a closed system, as the different interpretations seem to suggest; any reading of quantum mechanics must face the challenges posed by the complexity of the actual physical world at different scales, from the cosmological to the elementary particles. That raises the question of defining the relationship between the complex time and the polarized vacuum in ordinary quantum field theory. It is clear that while in quantum field theory actual physical properties are re-scaled according to the equations of the renormalization semi-group, localization requires instead a defined skeleton mass. One possibility would be simply to assign the task of describing the interaction vertices with a unitary formalism to the quantum field theory, and to extend the model under consideration to the asymptotic states (obviously, a no-rescalable notion). However, the discussion of this delicate problem requires further reflections targeted to the formal definition of a relationship between the two descriptions. At the present time we have to leave this question open. At a higher speculative level, the question of the Planck scale remains, considering that between this one and the typical range of quantum mechanics there is a gap of 16 orders of magnitude and 20 between the chronon and the Planck time. Eventually, even if quantum mechanics is revealed to be emergent, this will be in favor of a pre-space and purely algebraic theory of the foundations of physics.

\section{Acknowledgements}

The authors wish to thank Eliahu Cohen and John Ashmead for valuable suggestions that have improved the quality of this article. 


\section{References}

[1] Ghirardi G. Sneaking a Look at God's Cards: Unraveling the Mysteries of Quantum Mechanics. Malsbary G (translator), Princeton: Princeton University Press, 2007.

[2] Daneri A, Loinger A, Prosperi GM. Quantum theory of measurement and ergodicity conditions. Nuclear Physics 1962; 33: 297-319. doi:10.1016/ 0029-5582(62)90528-X

[3] Bohm D, Bub J. A proposed solution of the measurement problem in quantum mechanics by a hidden variable theory. Reviews of Modern Physics 1966; 38 (3): 453-469. doi : 10.1103/RevModPhys. 38. 453

[4] Longtin L, Mattuck RD. Relativistically covariant Bohm-Bub hidden-variable theory for spin measurement of a single particle. Foundations of Physics 1984; 14 (8): 685-703. doi:10.1007/ bf00736616

[5] Ghirardi GC, Rimini A, Weber T. Unified dynamics for microscopic and macroscopic systems. Physical Review D 1986; 34 (2): 470-491. doi:10.1103/ PhysRevD. 34.470

[6] Schlosshauer M. Decoherence, the measurement problem, and interpretations of quantum mechanics. Reviews of Modern Physics 2005; 76 (4): 1267-1305. arXiv:quant-ph/0312059, doi: 10 . 1103/RevModPhys.76.1267

[7] Bergquist JC, Hulet RG, Itano WM, Wineland DJ. Observation of quantum jumps in a single atom. Physical Review Letters 1986; 57 (14): 1699-1702. doi : 10.1103/PhysRevLett.57.1699

[8] Nagourney W, Sandberg J, Dehmelt H. Shelved optical electron amplifier: observation of quantum jumps. Physical Review Letters 1986; 56 (26): $2797-$ 2799. doi: 10.1103/PhysRevLett. 56.2797

[9] Sauter T, Neuhauser W, Blatt R, Toschek PE. Observation of quantum jumps. Physical Review Letters 1986; 57 (14): 1696-1698. doi:10.1103/ PhysRevLett.57.1696

[10] Basche T, Kummer S, Brauchle C. Direct spectroscopic observation of quantum jumps of a single molecule. Nature 1995; 373 (6510): 132-134. doi:10.1038/373132a0
[11] Gleyzes S, Kuhr S, Guerlin C, Bernu J, Deleglise S, Busk Hoff U, Brune M, Raimond J-M, Haroche S. Quantum jumps of light recording the birth and death of a photon in a cavity. Nature 2007; 446 (7133): 297-300. doi : 10.1038/nature05589

[12] Peil S, Gabrielse G. Observing the quantum limit of an electron cyclotron: QND measurements of quantum jumps between Fock states. Physical Review Letters 1999; 83 (7): 1287-1290. doi:10.1103/ PhysRevLett . 83.1287

[13] Yu Y, Zhu S-L, Sun G, Wen X, Dong N, Chen J, Wu P, Han S. Quantum jumps between macroscopic quantum states of a superconducting qubit coupled to a microscopic two-level system. Physical Review Letters 2008; 101 (15): 157001. doi:10.1103/ PhysRevLett. 101.157001

[14] Neumann P, Beck J, Steiner M, Rempp F, Fedder H, Hemmer PR, Wrachtrup J, Jelezko F. Singleshot readout of a single nuclear spin. Science 2010; 329 (5991): 542-544. doi:10.1126/science. 1189075

[15] Vamivakas AN, Lu CY, Matthiesen C, Zhao Y, Falt S, Badolato A, Atature M. Observation of spindependent quantum jumps via quantum dot resonance fluorescence. Nature 2010; 467 (7313): 297300. doi : 10.1038/nature09359

[16] Vijay R, Slichter DH, Siddiqi I. Observation of quantum jumps in a superconducting artificial atom. Physical Review Letters 2011; 106 (11): 110502. arXiv: 1009.2969, doi:10.1103/ PhysRevLett.106.110502

[17] Itano WM, Bergquist JC, Wineland DJ. Early observations of macroscopic quantum jumps in single atoms. International Journal of Mass Spectrometry 2015 ; 377: 403-409. doi:10.1016/j.ijms. 2014.07 .005

[18] Davies PCW. Particles do not exist. In: Quantum Theory of Gravity: Essays in Honor of the 60th Birthday of Bryce S. DeWitt. Christensen SM (editor), Bristol, England: Adam Hilger, 1984, pp.6677.

[19] Pessa E. The concept of particle in quantum field theory. In: Vision of Oneness. Licata I, Sakaji AJ (editors), Rome: Aracne, 2011, pp.13-40.

[20] Colosi D, Rovelli C. What is a particle? Classical and Quantum Gravity 2009; 26 (2): 025002. doi: 10.1088/0264-9381/26/2/025002 
[21] Heisenberg W. Die physikalischen Prinzipien der Quantentheorie. Leipzig: S. Hirzel, 1941.

[22] Chiatti L, Licata I. Relativity with respect to measurement: collapse and quantum events from Fock to Cramer. Systems 2014; 2 (4): 576-589. doi: $10.3390 /$ systems 2040576

[23] Hartle JB, Hawking SW. Wave function of the universe. Physical Review D 1983; 28 (12): 2960-2975. doi : 10.1103/PhysRevD . 28.2960

[24] Licata I, Chiatti L. The archaic universe: Big Bang, cosmological term and the quantum origin of time in projective cosmology. International Journal of Theoretical Physics 2009; 48 (4): 1003-1018. doi : 10.1007/s10773-008-9874-z

[25] Licata I, Chiatti L. Archaic universe and cosmological model: "Big-Bang" as nucleation by vacuum. International Journal of Theoretical Physics 2010; 49 (10): 2379-2402. arXiv:0808.1339, doi : $10.1007 / \mathrm{s} 10773-010-0424-0$

[26] Parisi G. Statistical Field Theory. Advanced Book Classics, Boulder: Westview Press, 1998.

[27] Denkmayr T, Geppert H, Sponar S, Lemmel H, Matzkin A, Tollaksen J, Hasegawa Y. Observation of a quantum Cheshire Cat in a matter-wave interferometer experiment. Nature Communications 2014; 5: 4492. doi : 10.1038/ncomms 5492

[28] Chiatti L. The transaction as a quantum concept. International Journal of Research and Reviews in Applied Sciences 2013; 16 (4): 28-47. arXiv: 1204.6636

[29] Zeh H-D. There are no quantum jumps, nor are there particles! Physics Letters A 1993; 172 (4): 189-192. doi : 10.1016/0375-9601(93)91005-P

[30] Chiatti L. Wave function structure and transactional interpretation. In: Waves and Particles in Light and Matter. van der Merwe A, Garuccio A (editors), Berlin: Springer, 1994, pp.181-187. doi: 10. 1007/978-1-4615-2550-9_15

[31] Chiatti L. Path integral and transactional interpretation. Foundations of Physics 1995; 25 (3): 481-490. doi : 10.1007/bf02059232

[32] Kastner RE. The Transactional Interpretation of Quantum Mechanics: The Reality of Possibility. Cambridge: Cambridge University Press, 2013.
[33] Licata I. Transaction and non locality in quantum field theory. EPJ Web of Conferences 2014; 70: 00039. doi:10.1051/epjconf/20147000039

[34] Cini M. Field quantization and wave particle duality. Annals of Physics 2003; 305 (2): 83-95. doi : 10. 1016/SO003-4916(03)00042-3

[35] Feynman RP, Vernon Jr FL. The theory of a general quantum system interacting with a linear dissipative system. Annals of Physics 1963; 24: 118-173. doi : 10.1016/0003-4916(63)90068-X

[36] Schwinger J. Brownian motion of a quantum oscillator. Journal of Mathematical Physics 1961; 2 (3): 407-432. doi : 10.1063/1.1703727

[37] Blasone M, Srivastava YN, Vitiello G, Widom A. Phase coherence in quantum Brownian motion. Annals of Physics 1998; 267 (1): 61-74. arXiv: quant-ph/9707048, doi:10.1006/aphy.1998. 5811

[38] Vitiello G. Classical trajectories and quantum field theory. Brazilian Journal of Physics 2005; 35 (2A): 351-358. doi : 10.1590/S0103-97332005000200021

[39] Caldirola $P$. The introduction of the chronon in the electron theory and a charged-lepton mass formula. Lettere al Nuovo Cimento 1980; 27 (8): 225-228. doi : $10.1007 / \mathrm{bf02750348}$

[40] Recami E, Olkhovsky VS, Maydanyuk SP. On non-self-adjoint operators for observables in quantum mechanics and quantum field theory. International Journal of Modern Physics A 2010; 25 (9): 1785-1818. arXiv:0903.3187, doi:10.1142/ S0217751X10048007

[41] Battey-Pratt EP, Racey TJ. Geometric model for fundamental particles. International Journal of Theoretical Physics 1980; 19 (6): 437-475. doi:10. 1007/BF00671608

[42] Rietdijk CW. The world is realistically fourdimensional, waves contain information embodied by particles codedly, and microphysics allows understandable models I. Annales de la Fondation Louis de Broglie 1988; 13 (2): 141-182.

[43] Rietdijk CW. The world is realistically fourdimensional, waves contain information embodied by particles codedly, and microphysics allows understandable models II. Annales de la Fondation Louis de Broglie 1988; 13 (3): 299-336. 
[44] Kleinert H. Hadronization of quark theories. In: Understanding the Fundamental Constituents of Matter, vol.14. The Subnuclear Series, Zichichi A (editor), New York: Plenum Publishing, 1978, pp.289-389. doi : 10.1007/978-1-4684-0931-4_7

[45] Hagedorn R. Statistical thermodynamics of strong interactions at high energies. Supplemento al Nuovo Cimento 1965; 3 (2): 147-186. CERN: 938671

[46] Hagedorn R, Rafelski J. From hadron gas to quark matter I. In: Statistical Mechanics of Quarks and Hadrons. Satz H (editor), Amsterdam: North Holland, 1981, pp.237-251. CERN: 125482

[47] Rafelski J, Hagedorn R. From hadron gas to quark matter II. In: Statistical Mechanics of Quarks and Hadrons. Satz H (editor), Amsterdam: North Holland, 1981, pp.253-272. CERN: 126179

[48] Chiatti L, Licata I. Quark gluon plasma as a critical state of de Sitter geometries. 2015, doi:10.13140/ 2.1.1181.2167

[49] Ashman J, et al. A measurement of the spin asymmetry and determination of the structure function $g_{1}$ in deep inelastic muon-proton scattering. Physics Letters B 1988; 206 (2): 364-370. doi: 10.1016/ 0370-2693(88)91523-7

[50] Prok Y, et al. Precision measurements of $g_{1}$ of the proton and of the deuteron with $6 \mathrm{GeV}$ electrons. Physical Review C 2014; 90 (2): 025212. doi : 10. 1103/PhysRevC.90.025212

[51] Penrose R. The Road to Reality: A Complete Guide to the Laws of the Universe. London: Jonathan Cape, 2004.

[52] Cini M, De Maria M, Mattioli G, Nicolò F. Wave packet reduction in quantum mechanics: a model of a measuring apparatus. Foundations of Physics 1979; 9 (7-8): 479-500. doi:10.1007/ bf00708364

[53] Zeh H-D. On the interpretation of measurements in quantum theory. Foundations of Physics 1970; 1 (1): 69-76. doi:10.1007/bf00708656
[54] Zurek WH. Decoherence and the transition from quantum to classical. Physics Today 1991; 44 (10): 36-44. doi: 10.1063/1.881293

[55] Aharonov Y, Bergmann PG, Lebowitz JL. Time symmetry in the quantum process of measurement. Physical Review B 1964; 134 (6): 1410-1416. doi:10.1103/PhysRev.134.B1410

[56] Aharonov Y, Albert DZ, Casher A, Vaidman L. Surprising quantum effects. Physics Letters A 1987; 124 (4): 199-203. doi : 10.1016/0375-9601(87) 90619-()

[57] Aharonov Y, Vaidman L. The two-state vector formalism: an updated review. In: Time in Quantum Mechanics. Lecture Notes in Physics, vol.734, Muga JG, Mayato RS, Egusquiza ÍL (editors), Springer, 2008, pp.399-447. doi : 10.1007/ 978-3-540-73473-4_13

[58] Elitzur AC, Cohen E. The retrocausal nature of quantum measurement revealed by partial and weak measurements. AIP Conference Proceedings 2011; 1408 (1): 120-131. doi:10.1063/1.3663720

[59] Bitbol M. Schrödinger's Philosophy of Quantum Mechanics. Boston Studies in the Philosophy and History of Science, vol.188, Berlin: Springer, 1996. doi : 10.1007/978-94-009-1772-9

[60] Bohm D, Hiley BJ. The Undivided Universe: An Ontological Interpretation of Quantum Theory. London: Routledge, 1993.

[61] Vértesi T, Brunner N. Quantum nonlocality does not imply entanglement distillability. Physical Review Letters 2012; 108 (3): 030403. arXiv: 1106.4850, doi:10.1103/PhysRevLett. 108.030403

[62] Buscemi F. All entangled quantum states are nonlocal. Physical Review Letters 2012; 108 (20): 200401. arXiv:1106.6095, doi:10.1103/ PhysRevLett.108.200401

[63] Fiscaletti D, Licata I. Bell length in the entanglement geometry. International Journal of Theoretical Physics 2015; 54 (7): 2362-2381. doi: 10.1007/s10773-014-2461-6 